\title{
LIGOPHORUS PARVICIRRUS n. sp. (MONOGENEA, ANCYROCEPHALIDAE) \\ parasite de Liza ramada (Risso, 1826) (Teleostei, Mugilidae)
}

\author{
L. EUZET* et D. SANFILIPPO
}

RÉSUMÉ. Description et position systématique de Ligophorus parvicirrus, nouveau parasite branchial de Liza ramada. Révision de la diagnose du genre Ligophorus Euzet et Suriano, 1977. Étude de la répartition branchiale et définition du microhabitat de Ligophorus parvicirrus. Mise en évidence des effets d'une compétition entre cette espèce et Ligophorus imitans Euzet et Suriano, 1977 autre parasite branchial de L. ramada.

\section{Ligophorus parvicirrus n. sp. (Monogenea, Ancyrocephalidae) parasite of Liza ramada (Risso, 1826) (Teleostei, Mugilidae).}

SUMMARY. Ligophorus parvicirrus, a new gill parasite of Liza ramada is deseribed with its taxonomic position. The diagnosis of the genus Ligophorus Euzet and Suriano, 1977 is reviewed. The branchial distribution of Ligophorus parvicirrus is given and its microhabitat defined. The effects of a competition between this species and Ligophorus imitans Euzet and Suriano, 1977, another gill parasite of L. ramada are shown.

En 1977, Euzet et Suriano ont décrit plusieurs Monogènes Ancyrocephalidae nouveaux, parasites branchiaux des Muges de Méditerranée occidentale. Jusqu'alors presque toutes ces espèces étaient confondues et nommées Ancyrocephalus vanbenedenii (Parona et Perugia, 1890). Euzet et Suriano ont montré qu'il existait en réalité 11 espèces différentes pour lesquelles ils ont créé le genre Ligophorus. La spécificité de ces Monogènes par rapport au poisson hôte est du type oioxène, d'après la définition de Euzet et Combes (1980).

Nous décrivons une nouvelle espèce de Ligophorus récoltée chez Liza ramada (Risso, 1826). Nous étudions la répartition de cet Ancyrocephalidae sur les branchies de l'hôte et mettons en évidence les effets d'une compétition entre deux espèces congénériques pour l'occupation de la surface branchiale.

* Laboratoire de Parasitologie Comparée, U.S.T.L., place E. Bataillon, F 34060 Montpellier Cedex. Laboratoire associé au C.N.R.S. (E.R.A.915).

Accepté le $I_{7}$ janvier $\mathrm{I}_{98} 8$. 


\section{Ligophorus parvicirrus n. sp.}

Hôte $\quad$ : Liza ramada (Risso, 1826) Mugilidae

Habitat : Branchies

Localité : Golfe du Lion

Matériel étudié : 50 individus sur le vivant

5 colorés et montés in toto. Cotypes lames TJ 119 (129 TC MNHN) ; TJ 120 (130 TC MNHN).

Les Vers adultes, à corps allongé assez étroit, mesurent de 0,8 à $1 \mathrm{~mm}$ de longueur et de 0,20 à $0,25 \mathrm{~mm}$ de largeur au niveau de l'ovaire. A l'extrémité antérieure débouchent, de chaque côté, trois groupes de glandes adhésives. Dans cette région céphalique on distingue deux paires de taches oculaires dorsales, celles de la paire postérieure, les plus grosses, sont munies d'un cristallin sphérique (fig. 1).

Le hapteur postérieur est armé de 14 crochetons marginaux, 2 paires d'hamuli et 2 barres transversales.

Les hamuli dorsaux ( $f g .2 \mathrm{D}$ ) ont une lame courbe, coudée à angle droit près de l'extrémité ; la pointe représente ainsi le quart de la lame. Les dimensions de ces hamuli, exprimées en $\mu \mathrm{m}$, sont :

$\mathrm{a}=40-45 ; \mathrm{b}=30-35 ; \mathrm{c}=10-12 ; \mathrm{d}=15-17 ; \mathrm{e}=8-9$

Entre ces hamuli se place une barre transversale dorsale en $\mathrm{V}$ très ouvert. Cette barre mesure 32 à $35 \mu \mathrm{m}$ de longueur (fig. $2 \mathrm{D}$ ).

Les hamuli ventraux ( fig. $2 \mathrm{~V}$ ) ont une morphologie très voisine de celle dés dorsaux. Le manche présente dans l'encoche, une légère excroissance. Ces hamuli ventraux mesurent en $\mu \mathrm{m}$ :

$\mathrm{a}=41-45 ; \mathrm{b}=33-35 ; \mathrm{c}=9-10 ; \mathrm{d}=15-17 ; \mathrm{e}=8-9$

La barre transversale ventrale porte deux petites expansions antérieures symétriques distantes de $8 \mu \mathrm{m}$. La barre mesure 35-38 $\mu \mathrm{m}$ de longueur ( $f$ g. $2 \mathrm{~V}$ ).

Les 14 crochetons marginaux du hapteur ont 12 à $15 \mu \mathrm{m}$ de longueur. Ils ont tous la même morphologie avec un manche droit de 10-12 $\mu \mathrm{m}$, une courte garde et une lame arquée. Ils sont disposés symétriquement de chaque côté du hapteur, selon l'arrangement caractéristique déjà décrit chez plusieurs Ancyrocephalidae. De chaque côté, on a :

-5 crochetons ventraux dont 3 latéraux et 2 plus centraux (l'un près de la lame de l'hamuli ventral, l'autre en avant de la barre transversale).

-2 crochetons dorsaux et latéraux.

A la bouche subterminale antérieure fait suite un pharynx musculeux mesurant $50 \mu \mathrm{m}$ de longueur et $30 \mu \mathrm{m}$ dans son plus grand diamètre. L'œsophage, très court, bifurque en deux branches intestinales simples qui se rejoignent postérieurement en formant un anneau complet (fig. 1).

Le testicule médian est situé entre les branches intestinales, dans la moitié postérieure du corps. Le canal déférent, légèrement sinueux, remonte dorsalement sur le côté gauche sans entourer la branche intestinale. Il aboutit à la base d'une 

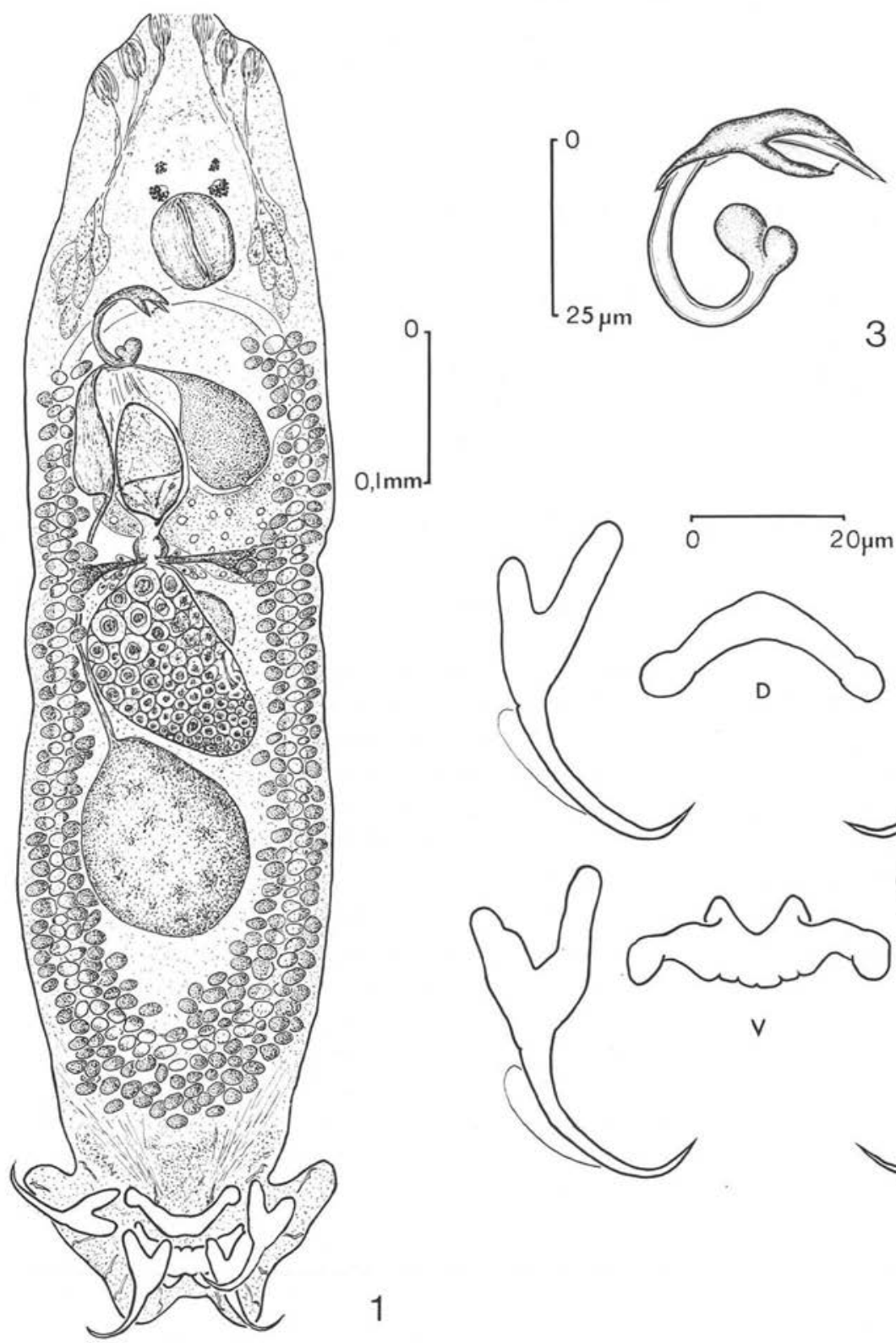

$0,1 \mathrm{~mm}$
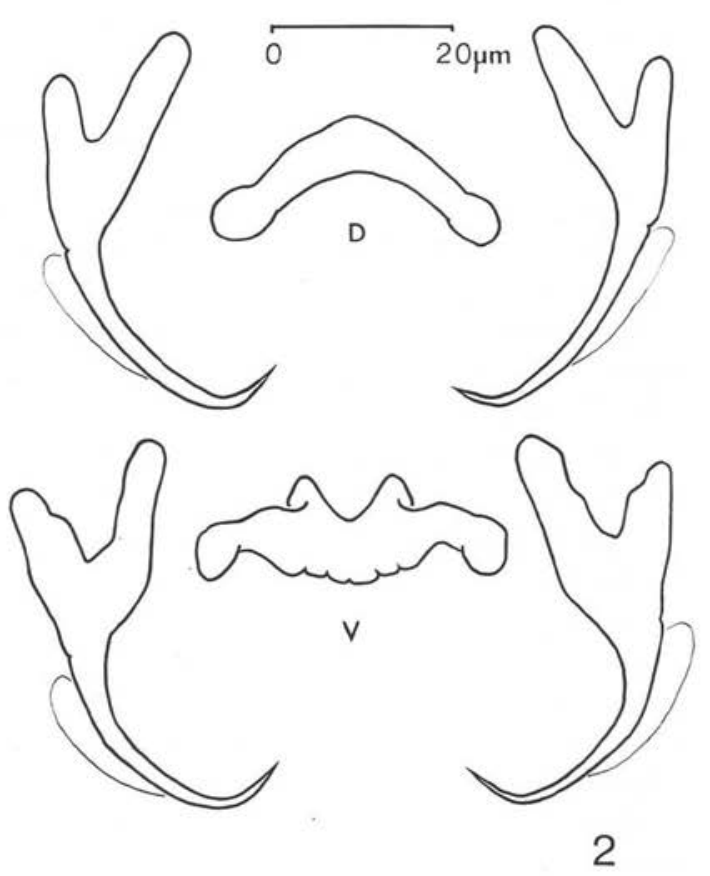

Fig. I. - Ligophorus parvicirrus n. sp. Animal in toto, en vue dorsale.

FIG. 2. - Ligophorus parvicirrus. Éléments sclérifiés du hapteur.

D : Barre transversale et hamuli dorsaux ; V : Barre transversale et hamuli ventraux.

FIG. 3. - Ligophorus parvicirrus. Cirre et pièce accessoire. 
vésicule séminale fusiforme de 40 à $50 \mu \mathrm{m}$ de longueur. Cette vésicule vient déboucher par un fin canal à la base du cirre. A ce niveau se déverse aussi le canal d'un réservoir prostatique piriforme, long de $40 \mu \mathrm{m}$. Ce réservoir reçoit à son extrémité postérieure les sécrétions d'une glande qui paraît plurinuclée (fig. 1).

L'appareil copulateur se compose d'un petit cirre médian et d'une pièce accessoire. Le cirre tubulaire, long de 60 à $80 \mu \mathrm{m}$, forme un tour de spire. L'extrémité libre peut jouer dans une pièce accessoire arquée, élargie distalement et mesurant $30 \mu \mathrm{m}$ environ ( fig. 3).

L'ovaire globuleux intercœcal est situé immédiatement en avant du testicule. Le réceptable séminal ventral reçoit postérieurement un mince canal vaginal souvent difficile à observer car les parois ne sont pas sclérifiées comme chez les autres Ligophorus. L'ouverture du vagin est située à mi-hauteur du corps, légèrement à droite du plan médio-sagittal.

Les œufs ovoïdes mesurent $30 \mu \mathrm{m}$ de longueur et $20 \mu \mathrm{m}$ de diamètre. Le pôle opposé à l'opercule est marqué par un petit mucron.

\section{Discussion}

Les caractéristiques de ce Monogène, en particulier le nombre et la disposition des pièces sclérifiées du hapteur, les deux branches intestinales unies postérieurement, le canal déférent gauche n'entourant pas la branche intestinale, l'ovaire intercœeal, le vagin ventral sub-médian droit, permettent de le classer dans le genre Ligophorus Euzet et Suriano, 1977. Mais, d'après ces auteurs, le genre est défini comme ayant " un cirre tubulaire long et mince » et " un vagin sclérifié sub-médian droit ».

En fonction de la description du parasite nouveau de Liza ramada, nous proposons de modifier la diagnose générique de la manière suivante :

Genre Ligophorus Euzet et Suriano, 1977, Ancyrocephalidae. Hapteur armé de 14 crochetons, 2 paires d'hamuli réunis par deux barres transversales (1 dorsale, 1 ventrale). Trois paires d'amas glandulaires céphaliques. Quatre ocelles dorsaux prépharyngiens. Branches intestinales simples, unies postérieurement. Testicule médian. Canal déférent gauche. Vésicule séminale présente. Un réservoir prostatique. Cirre tubulaire mince avec une pièce accessoire. Ovaire médian prétesticulaire. Vagin sclérifié ou non à ouverture ventrale sub-médiane droite. Réceptacle séminal ventral en avant de l'ovaire. Euf operculé avec une ébauche de filament au pôle opposé à l'opercule. Parasite de Mugilidae. Type du genre : Ligophorus vanbenedenii (Parona et Perugia, 1890).

La taille du cirre, la morphologie de la pièce accessoire, l'absence de sclérification du vagin, la morphologie et la taille des pièces du hapteur, permettent de différencier le parasite décrit des onze espèces placées par Euzet et Suriano dans le genre Ligophorus.

Nous considérons cette espèce comme nouvelle et proposons de la nommer Ligophorus parvicirrus n. sp. pour marquer la taille caractéristique du cirre. 
Outre Ligophorus parvicirrus, deux autres Monogèens branchiaux, Ligophorus imitans Euzet et Suriano, 1977 et Ligophorus confusus Euzet et Suriano, 1977 sont des parasites spécifiques de Liza ramada. Mais les trois espèces ne se rencontrent pas chez cet hôte avec la même prévalence. Chez 103 L. ramada examinés au cours de cette étude, la prévalence est de $75 \%$ pour Ligophorus imitans, $42 \%$ pour L. parvicirrus et $4 \%$ seulement pour $L$. confusus.

Ces chiffres ne traduisent cependant que de façon partielle le parasitisme. Les poissons étudiés ont été en effet pêchés dans trois régions distinctes :

1 - La Méditerranée, au large de Sète et du Grau-du-Roi ;

2 - le Bassin de Thau où la salinité de l'eau est voisine de celle de la mer, avec de légères variations saisonnières ;

3 - l'étang du Vaccarès, en Camargue, où la salinité de l'eau est toujours faible.

Dans les zones de pêche, elle a varié de 2 à $5 \%$.

Liza ramada est un poisson qui supporte très bien les eaux dessalées. Nous avons pu examiner trois individus pêchés dans le Rhône à Beaucaire où les eaux sont presque douces. Ces trois Muges ne présentaient pas de Ligophorus.

Nous indiquons (tableau I) la prévalence des 3 Ligophorus selon le lieu de capture du poisson-hôte.

TABLEAU I. - Prévalence des trois Ligophorus parasites de Liza ramada selon le lieu de capture de l'hôte dans le sud de la France.

\begin{tabular}{|c|c|c|c|c|c|c|c|}
\hline & \multirow{2}{*}{$\begin{array}{l}\text { Nombre de } \\
\text { Poissons } \\
\text { examinés }\end{array}$} & \multicolumn{2}{|c|}{ L. imitans } & \multicolumn{2}{|c|}{ L. parvicirrus } & \multicolumn{2}{|c|}{ L. confusus } \\
\hline & & $\mathrm{N}$ & Prév. & $\mathrm{N}$ & Prév. & $\mathrm{N}$. & Prév. \\
\hline Étang du Vaccarès & 78 & 63 & $81 \%$ & 28 & $36 \%$ & 0 & $0 \%$ \\
\hline Étang de Thau & 13 & 7 & $54 \%$ & 5 & $38 \%$ & 4 & $30 \%$ \\
\hline Golfe du Lion & 12 & 7 & $58 \%$ & 10 & $83 \%$ & 0 & $0 \%$ \\
\hline Total & 103 & 77 & $75 \%$ & 43 & $42 \%$ & 4 & $4 \%$ \\
\hline
\end{tabular}

$\mathrm{N}$ : Nombre de Poissons parasités par l'espèce indiquée.

Prév. : Prévalence du parasite.

Ainsi L. confusus n'a été observé que dans le Bassin de Thau. Le petit nombre d'individus récoltés n'a pas permis de déterminer leur répartition sur les branchies.

Afin de connaitre le microhabitat de $L$. imitans et $L$. parvicirrus, pour chaque poisson hôte nous avons noté, sur une représentation schématique du système branchial, la localisation des parasites récoltés. Ce schéma comprend une silhouette de l'hémibranchie antérieure et postérieure des quatre arcs branchiaux droits et gauches. Chaque hémibranchie est subdivisée en cinq secteurs numérotés de 1 à 5 depuis l'attachement dorsal jusqu'à la copula ventrale et en trois zones, nommées : basale, médiane et distale, depuis la base jusqu'à l'extrémité des filaments ( fig. 4). 


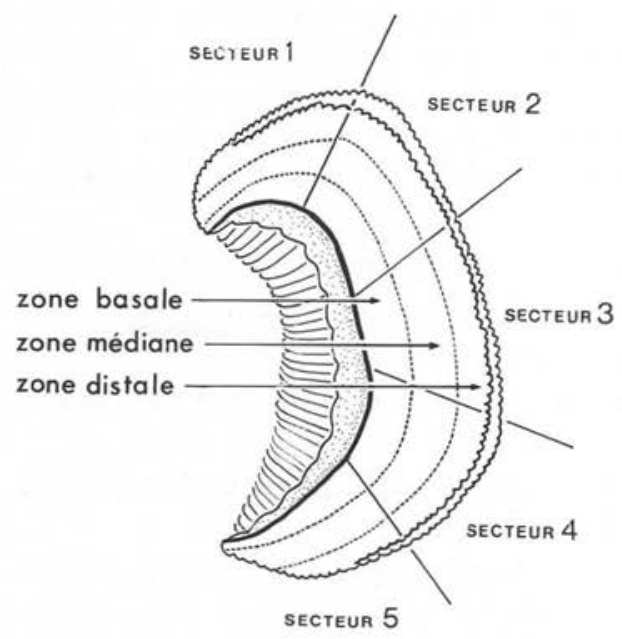

FIG. 4. - Délimitation des zones et des secteurs employés pour la localisation des Ligophorus sur les arcs branchiaux de Liza ramada.

\section{4}

Parmi les 75 L. ramada examinés pour cette étude, 40 n'étaient parasités que par Ligophorus imitans, 10 l'étaient par L. parvicirrus seul et 24 présentaient simultanément ces deux Monogènes.

\section{Infestations unispécifiques}

\section{Répartition par arc}

Nous résumons dans le tableau II, la répartition des deux Ligophorus sur les arcs branchiaux des poissons-hôtes. Les chiffres donnés représentent la somme des individus trouvés sur les arcs du côté droit et du côté gauche. Les chiffres prouvent, en effet, qu'il n'existe pas de différence significative entre les deux côtés du corps.

- Ligophorus imitans est réparti selon un gradient antéro-postérieur nettement décroissant (fig. 5).

- Ligophorus paricirrus occupe les quatre arcs avec cependant une légère préférence pour la quatrième.

TABLEAU/II. - Répartition selon les arcs de deux Ligophorus parasites de L. ramada.

\begin{tabular}{|c|c|c|c|c|c|c|c|c|}
\hline \multirow[b]{2}{*}{ L. imitans } & \multicolumn{2}{|r|}{ A I } & \multicolumn{2}{|c|}{ A II } & \multicolumn{2}{|c|}{ A III } & \multicolumn{2}{|c|}{ A IV } \\
\hline & 358 & $43,5 \%$ & 228 & $27 \%$ & 176 & $21 \%$ & 71 & $8,5 \%$ \\
\hline L. parvicirrus & 95 & $21 \%$ & 107 & $23 \%$ & 110 & $24 \%$ & 148 & $32 \%$ \\
\hline
\end{tabular}



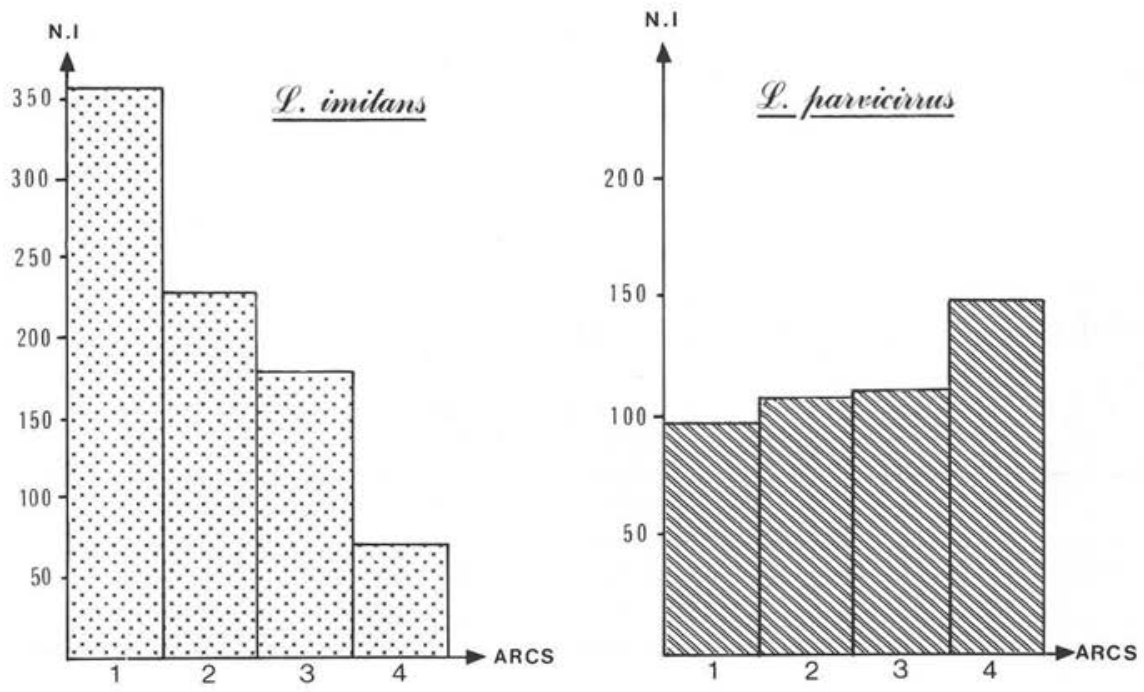

infestations unispécifiques
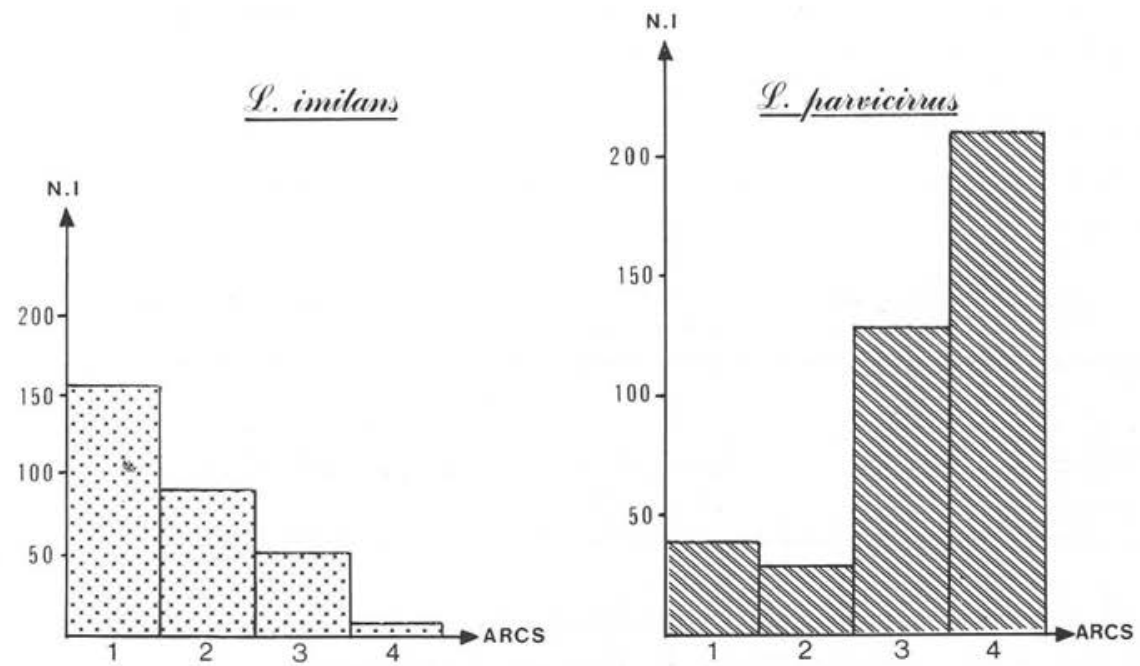

infestations simultanées

FIG. 5. - Répartition par arcs de Ligophorus imitans et Ligophorus parvicirrus, lors des infestations unispécifiques.

FIG. 6. - Répartition par arcs de Ligophorus imitans et Ligophorus parvicirrus, lors des infestations simultanées. 
Si l'on tient compte du fait que la surface offerte par les hémibranchies de ce quatrième arc est inférieure à celle des trois premiers, L. parvicirrus est réparti selon un gradient antéro-postérieur croissant (fig. 5).

\section{Répartition par zones}

Dans le tableau III, nous donnons les chiffres qui traduisent la répartition par zone des deux Ligophorus et le pourcentage que cela représente par rapport au nombre total d'individus récoltés pour chaque espèce.

TABLEAU III. - Répartition de deux Ligophorus selon les trois zones délimitées sur les branchies de $L$. ramada.

\begin{tabular}{lrrrrrrrr}
\hline & Zone basale & \multicolumn{3}{c}{ Zone médiane } & \multicolumn{2}{c}{ Zone distale } \\
\hline L. imitans & 523 & 63,5 & $\%$ & 297 & 35 & $\%$ & 13 & $1,5 \%$ \\
L.parvicirrus & 87 & 19 & $\%$ & 306 & 66,5 & $\%$ & 67 & $14,5 \%$ \\
\hline
\end{tabular}

L'optimum des deux espèces est distinct. L. imitans préfère la base des filaments branchiaux, tandis que $L$. parvicirrus est surtout accroché dans la zone médiane (fig. 7).

Notons que les quelques individus de Ligophorus confusus que nous avons récoltés semblent être localisés dans la zone distale des hémibranchies.

Répartition par secteur

Cette répartition dorso ventrale (tableau $I V$ ) est comparable pour les deux espèces :

TABLEAU IV. - Répartition de deux Ligophorus parasites de L. ramada, selon les 5 secteurs délimités sur les branchies.

\begin{tabular}{|c|c|c|c|c|c|c|c|c|c|c|}
\hline & 1 & & 2 & & 3 & & 4 & & 5 & \\
\hline L. imitans & 58 & $7 \%$ & 216 & $26 \%$ & 267 & $32 \%$ & 230 & $27,5 \%$ & 62 & $7,5 \%$ \\
\hline L. parvicirrus & 50 & $11 \%$ & 106 & $23 \%$ & 152 & $33 \%$ & 115 & $25 \%$ & 37 & $8 \%$ \\
\hline
\end{tabular}

Le secteur 3 représente un optimum de part et d'autre duquel les Ligophorus sont de moins en moins nombreux (fig. 8).

Ainsi, lors des infestations unispécifiques, Ligophorus imitans et Ligophorus parvicirrus occupent sur les branchies de $L$. ramada, un microhabitat relativement séparé. Si l'optimum selon les secteurs est commun, la répartition par zone et par arc, est différente. L. imitans préfère la zone basale, L. parvicirrus, la zone médiane. La répartition par arc fait apparaître que $L$. imitans est plutôt antérieur, L. parvicirrus plutôt postérieur. 

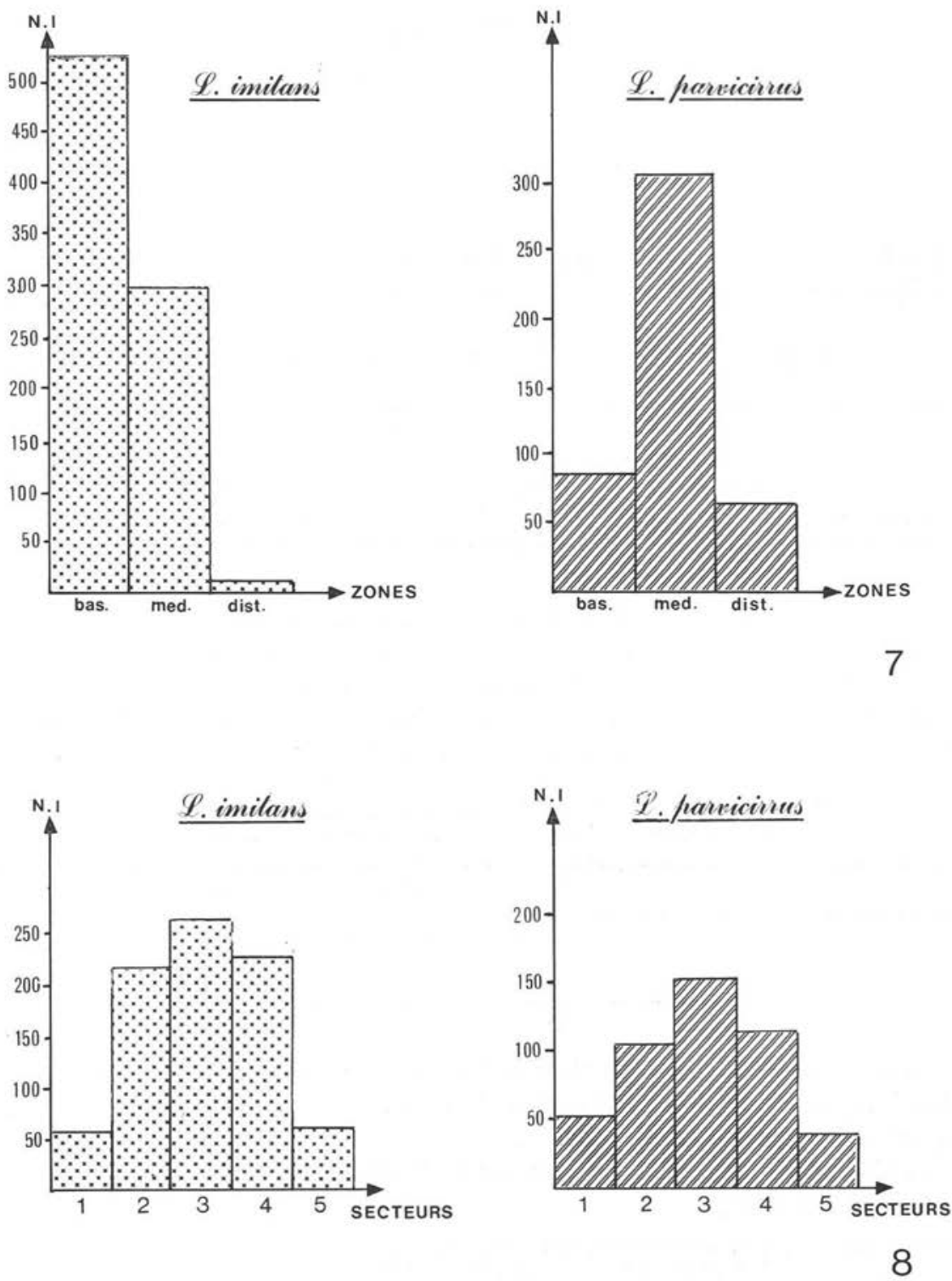

FIG. 7. - Répartition par zones de Ligophorus imitans et Ligophorus parvicirrus, lors des infestations unispécifiques.

FIG. 8. - Répartition par secteurs de Ligophorus imitans et Ligophorus parvicirrus, lors des infestations unispécifiques. 


\section{Infestations simultanées}

25 poissons sur 75 étaient parasités simultanément par L. imitans et L. parvicirrus.

\section{Répartition par arcs}

Nous indiquons, dans le tableau $V$, la répartition par arcs de Ligophorus imitans lors des infestations simultanées et, afin de faciliter les comparaisons, nous rappelons celle observée lors des infestations unispécifiques.

TABleaU V. - Répartition selon les arcs, chez L. ramada, de Ligophorus imitans seul et en présence de L. parvicirrus.

\begin{tabular}{lrrrrrrrrrr}
\hline & \multicolumn{2}{c}{ Arc I } & \multicolumn{2}{c}{ Arc II } & \multicolumn{2}{c}{ Arc III } & \multicolumn{2}{c}{ Arc IV } \\
\hline Infestations unispécifiques & 358 & 43,5 & $\%$ & 228 & 27 & $\%$ & 176 & $21 \%$ & 71 & $8,5 \%$ \\
Infestations simultanées & 154 & 51 & $\%$ & 87 & 28,5 & $\%$ & 51 & $17 \%$ & 11 & $3,5 \%$ \\
\hline
\end{tabular}

Entre les deux répartitions, la différence est significative. Il y a un léger déplacement de la population de $L$. imitans depuis les arcs III et IV, vers les arcs I et II lorsque ce Monogène est en présence de L. parvicirrus.

La répartition par arcs de Ligophorus parvicirrus récoltés lors des infestations unispécifiques et simultanées avec L. imitans, est résumée dans le tableau VI.

TABleAU VI. - Répartition selon les arcs, chez L. ramada, de Ligophorus parvicirrus seul et en présence de L. imitans.

\begin{tabular}{lrrrrrrrrrrr}
\hline & \multicolumn{2}{c}{ Arc I } & \multicolumn{2}{c}{ Arc II } & \multicolumn{2}{c}{ Arc III } & \multicolumn{2}{c}{ Arc IV } \\
\hline Infestations unispécifiques & 95 & 21 & $\%$ & 107 & 23 & $\%$ & 110 & 24 & $\%$ & 148 & $32 \%$ \\
Infestations simultanées & 39 & 9,5 & $\%$ & 30 & 7 & $\%$ & 127 & 31,5 & $\%$ & 210 & $52 \%$ \\
\hline
\end{tabular}

La différence entre les deux répartitions est très significative. La distribution de L. parvicirrus est modifiée par la présence de L. imitans. L. parvicirrus tend à se localiser sur les arcs postérieurs ( fig. 6).

La répartition par zone et par secteur ne subit pas de changements significatifs (tableaux VII et VIII).

TABLEAU VII. - Répartition branchiale par zone chez L. ramada, de Ligophorus imitans et L. parvicirrus, lors des infestations simultanées

\begin{tabular}{lcccccc}
\hline & Zone basale & \multicolumn{2}{c}{ Zone médiane } & \multicolumn{2}{c}{ Zone distale } \\
\hline L. imitans & 193 & $64 \%$ & 100 & $33 \%$ & 10 & $3 \%$ \\
L. parvicirrus & 38 & $20 \%$ & 274 & $68 \%$ & 49 & $12 \%$ \\
\hline
\end{tabular}


TABLEAU VIII - Répartition branchiale par secteur chez L. vamada et $L$. parvicirrus lors d'infestations simultanées par les deux espèces.

\begin{tabular}{lllllllllrr}
\hline & \multicolumn{1}{l}{1} & \multicolumn{2}{l}{2} & \multicolumn{2}{l}{3} & & 4 & \multicolumn{2}{c}{5} \\
\hline L. imitans & 18 & $6 \%$ & 82 & $27 \%$ & 98 & $32 \%$ & 81 & $27 \%$ & 24 & $8 \%$ \\
L. parvicirrus & 37 & $9 \%$ & 80 & $22 \%$ & 142 & $35 \%$ & 97 & $24 \%$ & 41 & $10 \%$ \\
\hline
\end{tabular}

La comparaison des tableaux VII et VIII avec les tableaux III et IV prouve que les deux parasites conservent dans leur répartition, le même optimum que lors des infestations unispécifiques.

Il apparaît cependant que lorsque deux espèces de Ligophorus se rencontrent chez un même individu hôte, si leur localisation sur les branchies ne varie pas, leur répartition sur les différents arcs est modifiée.

Nous estimons que ces modifications représentent le résultat d'une compétition entre les deux parasites. La séparation du microhabitat branchial selon les zones, que nous interprétons comme le premier résultat de cette compétition, ne paraît pas suffisante. Les deux Ligophorus tendent à séparer encore plus leur niche écologique en se cantonnant préférentiellement sur les divers arcs. L. imitans, en occupant surtout les arcs I et II, tend à repousser $L$. parvicirrus sur les arcs III et IV. Cette espèce, lorsqu'elle est seule, est présente sur tous les arcs.

La répartition notée représente donc un état d'équilibre entre les deux parasites.

Il faudrait maintenant connaître l'influence sur cet équilibre, de la troisième espèce, L. confusus, parasite oioxène de L. ramada et surtout expérimenter pour juger les effets de l'importance quantitative des deux populations.

Nous devons ajouter que dans le Golfe du Lion, Liza ramada est parasitée par un autre Ancyrocephalidae très voisin de Ligophorus, Ergenstrema mugilis Paperna, 1964 Monogène récolté surtout sur les branchiospines (Lambert et Sanfilippo, 1977). Ainsi, le microhabitat étant très différent Ergenstrema n'est pas en compétition avec les Ligophorus fixés sur les filaments branchiaux.

\section{BIBLIOGRAPHIE}

Bychovskaya-PAvloskaya I. E. et coll. : Key to Parasites of freshwater fish of the USSR. Akad. Nauk. S.S.S.R., I962, 9 I9 p. (traduit du russe par IBST Jérusalem, 1964).

Euzer L. : Parasitisme branchial simultané par deux espèces congénériques de Monogènes Monopisthocotylea. C.R. I I Multicolloque Européen Parasitologie, Rennes I-4 sept. I971, I972, 76-77.

Euzet L., Suriano D. M. : Ligophorus n. g. (Monogenea : Ancyrocephalidae) parasites des Mugilidae (Téléostéens) en Méditerranée. Bull. Mus. Nat. Hist. Nat., Paris, $3^{\text {e }}$ sér., no $47^{2}$, Zool., 329, $1977,797-822$.

Euzet L., Combes C. : Les problèmes de l'espèce chez les animaux parasites, in Les Problèmes de l'espèce dans le règne animal. Mem. Soc. Zool. France, 1980, 40, 239-285.

LAMBERT A., MAILlARD C. : Répartition branchiale de deux Monogènes : Diplectanum aequans (Wagener, 1857) Diesing, 1858 et Diplectanum laubieri Lambert et Maillard, 1974 (Monogenea, Monopisthocotylea) parasites simultanés de Dicentrarchus labrax (Téléostéen). Ann. Parasitol. Hum. Comp., 1975, 50, 691-699.

LAmbert A., SANFILIPPo D. : Position systématique et biologie de Ergenstrema mugilis Paperna, I964 (Monogenea, Monopisthocotylea) parasite de Liza (Liza) ramada (Risso, I826) (Téléos-

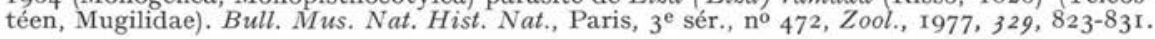

